\title{
Fast mechanism-based emulator of a slow urban hydrodynamic drainage simulator
}

\author{
David Machac ${ }^{\mathrm{a}, \mathrm{b}, *}$, Peter Reichert ${ }^{\mathrm{a}, \mathrm{b}}$, Jörg Rieckermann ${ }^{\mathrm{a}}$, Carlo Albert ${ }^{\mathrm{a}}$ \\ ${ }^{a}$ Eawag, Swiss Federal Institute of Aquatic Science and Technology, Department of Systems \\ Analysis, Integrated Assessment and Modelling, 8600 Dübendorf, Switzerland \\ ${ }^{b}$ ETH Zurich, Department of Environmental Systems Science, 8092 Zurich, Switzerland
}

\begin{abstract}
Gaussian process (GP) emulation is a data-driven method that substitutes a slow simulator with a stochastic approximation. It is then typically orders of anitude faster than the simulator at the costs of introducing interpolation errors. Our approach, the mechanism-based GP emulator, uses knowledge of the simulator mechanisms in addition to the information gained from previous simulator runs, so called design data. In this study, we investigate how the degree of incorporating mechanisms into the design of the GP emulator influences emulation accuracy. Similarly to the previous results, we get a significant gain in accuracy already when using the simplest approximation of the mechanisms by a single linear reservoir. However, in this case, we again considerably improve emulation accuracy when using the next two approximations. This allows us to decreases the required number of design data to achieve a similar accuracy as a 20 non-mechanistic emulator.
\end{abstract} 10

Keywords: emulation, Gaussian process, surrogate modelling, hydrological models

\section{Highlights:}

- We substitute a hydrological model for an urban catchment with a mechanism-

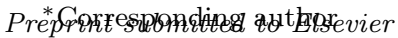

Email address: david.machac@eawag.ch (David Machac) 


\section{Introduction}

The sophistication of urban drainage simulators increases parallel to the available computational power. Hydrological simulators were considered computationally expensive two decades ago (Stieglitz et al., 1999; Axworthy and

40 Karney, 1999) and they still are today (Dobler and Pappenberger, 2013). They numerically solve (usually large) systems of partial differential equations of surface runoff and water flow in the sewer system. Even though new numerical techniques to handle this task more efficiently emerge constantly (e.g. Dongarra et al. (2014)), these efficiency gains are compensated by the demand for more 45 accurate and more detailed hydrological models. Computational speed becomes a limiting factor should we need to run a hydrological model tens of thousands of times, e.g. for the purpose of its calibration, for sensitivity analysis, or for uncertainty propagation. We can circumvent this problem by using a different model, which produces approximately the same results, but is orders of mag-

50 nitude faster. Such a model is called a surrogate model. The use of such a surrogate should always be followed by runs of the original simulator to validate the credibility of the results and, potentially, increase their accuracy.

The simplest techniques to build surrogate models is to build a lower-fidelity model of a high-fidelity simulator, by simplifying the original model or by re-

55 ducing the accuracy of its numerical solution. An example of the application of the former concept is given by Vanrolleghem et al. (2005), examples of the latter concept are the multiscale finite volume technique developed by Lunati and Jenny (2008) or the simulator by Forrester et al. (2006). A comprehensive overview of model-reducing algorithms used to achieve better performance is given by Gugercin and Antoulas (2004).

Another family of surrogate models are data-driven surrogates, where data in the name refers to pairs of inputs/outputs of the full simulator, so-called design data. These surrogates are more universal as they do not need to consider the structure of the model underlying the simulator. On the other hand,

65 these surrogates suffer from the curse of dimensionality regarding the number of simulator parameters (Bellman, 1956; Asher et al., 2015) by requiring strongly increasing sizes of the set of design data with increasing dimension of the parameter space. Due to the consideration of the model structure, lower-fidelity models do not need any design data and are therefore somewhat less sensitive 70 to this problem.

Data-driven surrogate modelling encompasses many different, well-known, methods, such as Kriging (Cressie, 1990), artificial neural networks (ANN) (Zurada, 1992) or polynomial chaos expansion (Sudret, 2008). Of these techniques, especially ANN gained some popularity in urban drainage modelling (Giustolisi 75 and Laucelli, 2005).

A comprehensive overview of surrogate modelling techniques, both lower fidelity and data-driven, in hydrology can be found in Razavi et al. (2012). This overview, however, shows that most articles about these methods do not include analytical estimates or measured results of the dependence of computation time on the size of the design data set, model complexity, and output dimension. 
A particularly well-known class of data-driven surrogate models, called Gaussian process (GP) emulators (Kennedy and O'Hagan, 2001; O'Hagan, 2006), are based on formulating a prior as a Gaussian stochastic process and then conditioning this process on the design data to construct the emulator as the 85 posterior. The emulator is then a statistical approximation of the simulator and provides uncertainty estimates in addition to the best estimate. To build surrogate models for dynamic simulators that produce time-series of results, we need to extend this basic emulation concept. The naïve technique of adding time as another input is not only inefficient, but can also lead to computational issues 90 when the time points are densely spaced. This led to the development of new methods that were specifically designed to emulate dynamic simulators (Conti et al., 2009; Higdon et al., 2008; Bayarri et al., 2007; Bhattacharya, 2007; Castelletti et al., 2012). The idea underlying one of these approaches (Liu and West, 2009), is to formulate a linear state-space model and use a Gaussian process as

95 a function of simulator parameters to represent the noise terms of replications of this model for design data sets. Conditioning this dynamic stochastic model with all design data sets then leads to the dynamic emulator.

Reichert et al. (2011) and Albert (2012) proposed to combine the advantages of low-fidelity surrogate models that use the knowledge of the model structure of 100 the simulator with those of data-driven approaches in the form of GP emulation. Their concept is to consider the mechanistic knowledge about the simulator by formulating a stochastic, linear state-space model as a simplified version of the simulator, formulate the noise term of replicate models as a Gaussian Process in the parameters, and condition the resulting stochastic model to the design 105 data. The idea of combining the two approaches is similar to the multi-fidelity surrogate modeling approach Leary et al. (2003); Forrester et al. (2007), but its implementation uses different concepts.

We showed the benefits of considering mechanisms for increasing emulator accuracy for a didactical example of flow through a single channel relying on 110 different numerical approximations of linearized open channel flow equations (Machac et al., 2015). The goal of this paper is to extend these results to an analysis of different spatial simplifications of an urban drainage simulation model (the Storm Water Management Model, $S W M M$ ) of a real catchment. We focus on emulation of a small number of outputs with respect to the simulator 115 parameters, such as a joint factor to the Manning-Strickler coefficients of the sewer pipes, and not on emulating different input time-series to the modelled system. This is what typically would be required for calibration or sensitivity analysis. Results at the full spatial resolution of the network could then be obtained by running the full simulator with the calibrated parameters. From 120 the conceptual point of view, emulating input time-series could be done similarly, however, this may result in practical difficulties due to the large expansion of the dimensionality of the parameter space. We also analyze the dependence of the gain in simulation time and, in particular, its dependence on the size of the design data set. 


\section{Case study and urban drainage simulator}

The future application we have in mind, for our emulator, is the calibration of the parameters of a SWMM model to a few measured time-series. These time-series are comprised of pairs of rainfall events and measured outflows of a catchment with each of these time-series being several hours long. To test the adequacy of the emulator for such a setting, we use a synthetic rainfall that excites the storage tank and makes the response strongly nonlinear. This means that the conditioning process of the stochastic, linear model on which the emulator is based, will be very important to get a good approximation to of the simulator response by the emulator. This is important for the test of different 135 emulators based on different simplifying models. On the other hand, using the synthetic input means that we do not have observed data. In this paper we will thus focus on quantifying the emulation accuracy as a function of the size of the design data set and the approximating linear model on which the emulator is based.

\subsection{Adliswil catchment}

We focus on a part of the urban drainage system of the city of Adliswil in the canton of Zurich, Switzerland, spanning an area of 162.8 ha. An overview of the situation is shown in Figure 1. We investigate the outflow at a wastewater treatment plant (WWTP), which then discharges into the river Sihl. Secondarily, we investigate water depth in a particular sewer manhole located approximately in the middle of the modelled area.

In order to investigate the influence of the topology of the catchment on the emulator, which is explained in detail later, we divide the catchment into two parts. The northern part (area of 93.69 ha) has a mainly pervious surface 150 (grass etc.) whereas the southern part (area of $69.10 \mathrm{ha}$ ) is more urban. This results in different response times for each part. The catchment also contains two combined sewer overflows and one storage tank (located in the southern part) with a complicated set of control rules (more than 80).

\section{2. $S W M M$ model}

Our SWMM model of the Adliswil catchment has 244 subcatchments and 460 conduits and was created from GIS data. The storage tank, which is non-linear due to its shape and control rules, is also modelled, albeit with a simplified set of rules. We generate an artificial rain event, as seen in Figure 2, which is strong enough to activate these non-linear elements in a short time. A simulation with this rain event of duration of 100 minutes with a one-minute time step takes approximately 3 seconds $^{1}$ on a CPU Intel Core i7-2600 CPU @ 3.40GHz.

\footnotetext{
${ }^{1}$ Although this seems like a short time that does not require an emulator, we have to keep in mind that it is just a test case on a small catchment spanning a short time period. Typically in an engineering practice, the simulation spans much longer periods. The aim of this work is to compare various simplified models and this comparison requires computationally intense parameter estimations, hence the short simulation time of 100 minutes.
} 


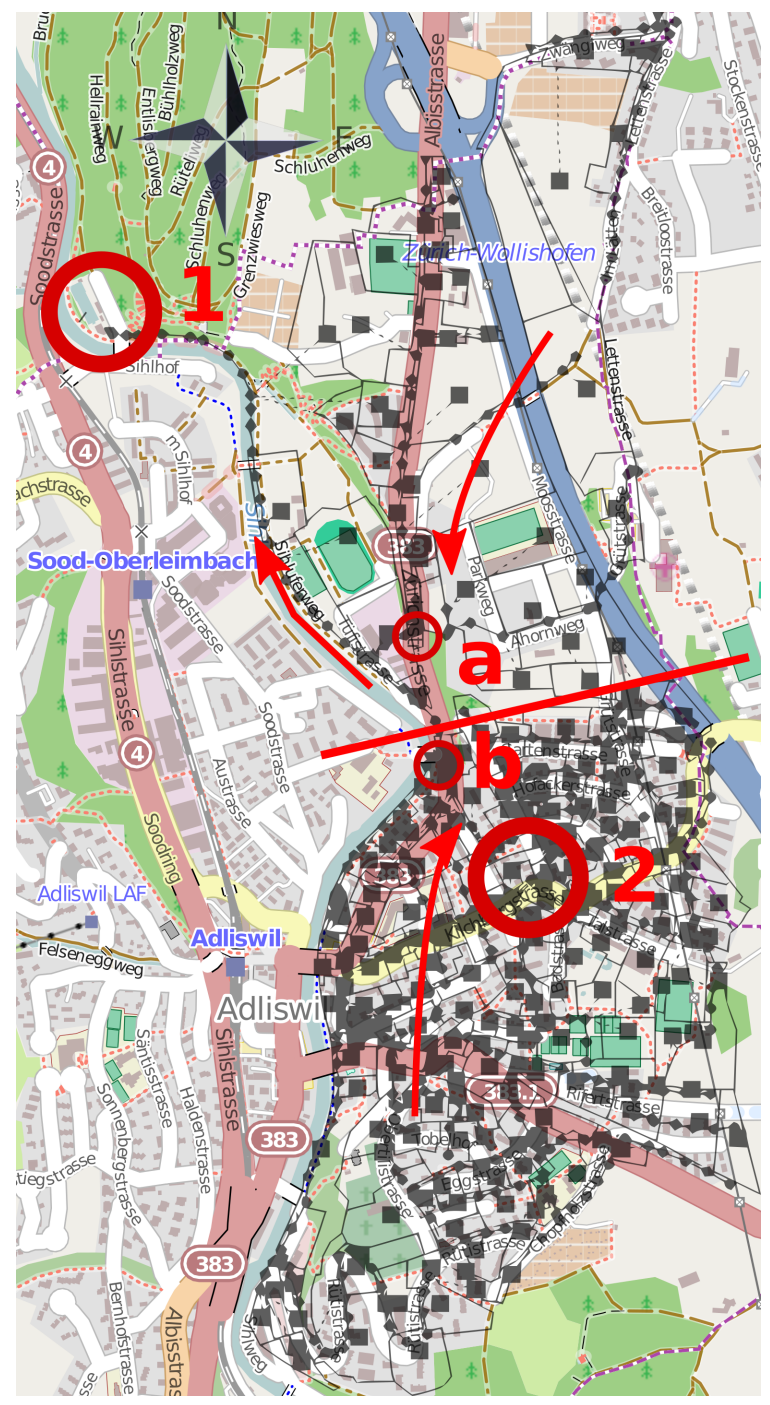

Figure 1: Overview of the modelled part of the urban drainage network of Adliswil with the overlaid SWMM model. Round black dots connected by solid black lines denote nodes connected by conduits, whereas black squares denote subcatchments. The outlet in the northwestern part of the catchment (red circle (1)), which discharges to the wastewater treatment plant (WWTP), is the outlet where we evaluate both the simulator and the emulator. The WWTP in turn discharges into the river Sihl, which flows in the northern direction. The red circle (2) in the southern part surrounds a manhole, where we observe the water depth (only for a multi-output emulation). The red line is a boundary between two parts of the catchment with different land use characteristics. Whereas the southern part is almost completely covered by streets and houses (impervious surfaces), in the northern part, grass surface is prevalent (pervious surface). The circles (a) and (b) denote the nodes used in Figure 2. Source of the map: openstreetmap.org, (c) OpenStreetMap contributors. 


\begin{tabular}{c|c|c|c|c|c|c} 
SWMM parameter class & Avg. value & Lowest & Highest & Unit & Abbrev. & Factor $\boldsymbol{\theta}$ \\
\hline \hline \% of impervious area per subcatchment & 36.00 & 9.16 & 92.09 & $\%$ & $r$ & $\theta_{1}$ \\
\hline characteristic width of overland flow path & 82.03 & 2.68 & 135.83 & $\mathrm{~m}$ & $w$ & $\theta_{2}$ \\
\hline slope of each subcatchment & 10.85 & 2.06 & 50.84 & $\%$ & $s$ & $\theta_{3}$ \\
\hline Manning's coefficient of the impervious area & 0.012 & 0.012 & 0.012 & $\mathrm{~s} \cdot \mathrm{m}^{-1 / 3}$ & $n_{\text {surf }}$ & $\theta_{4}$ \\
\hline \% of the impervious area without depression storage & 17.84 & 0 & 50 & $\%$ & - & $\theta_{5}$ \\
\hline height of depression storage on impervious areas & 2 & 2 & 2 & $\mathrm{~mm}$ & - & $\theta_{6}$ \\
\hline Manning's coefficient of the conduits & 0.01 & 0.01 & 0.01 & $\mathrm{~s} \cdot \mathrm{m}^{-1 / 3}$ & $n_{\text {net }}$ & $\theta_{7}$ \\
\hline \hline
\end{tabular}

Table 1: List of classes of catchment parameters. Only the scaling factors of these classes are varied. Averages that are weighted by the subcatchment areas and extremal values of these classes are shown for the whole catchment. The column with abbreviations shows parameters of the linear model underlying the emulator that are defined as functions of the associated parameter class.

The model contains thousands of parameters and it is infeasible to emulate with respect to all of them, as we would need to use a large design data set, which would in turn result in a slower emulator (this is explained later). However, a very large amount of parameters would also make calibration of SWMM infeasible due to parameter identifiability problems (Haag, 2006). Instead, we pick a parameter class of SWMM, e.g. all of the 460 roughness coefficients, and multiply those by a certain factor, common to all 460 occurrences. We then emulate with respect to this factor. The original values for these 460 roughness coefficients are estimated (Fu, 2013). In total, we have chosen 7 parameter classes to emulate (see Table 1). We denote a vector of parameter factors as $\boldsymbol{\theta}$ and we will confine its elements as

$$
0.5 \leq \theta_{i}^{\alpha} \leq 1.5, \quad \forall i \in\{1, \ldots, 7\} .
$$

We have chosen these particular parameter classes based on the sensitivity analysis carried out by Fu (2013). Because the model is highly sensitive to these 175 parameter classes, varying them allows us to simulate different regimes of the whole system, which is convenient for demonstrative purposes of the mechanismbased emulator. Also, emulating these parameters will be useful for our future work, as they cannot be inferred from the topography of the catchment and they have to be calibrated.

For a catchment of comparable size, the number of parameters calibrated in other case studies is usually between 5-15 (e.g. (Mancipe-Munoz et al., 2014)). Also, lumping parameters by using their common factors instead in order to reduce their number is a common practice (e.g. (Muleta and Boulos, 2008)), as it preserves the spatial variability of all subcatchments. 

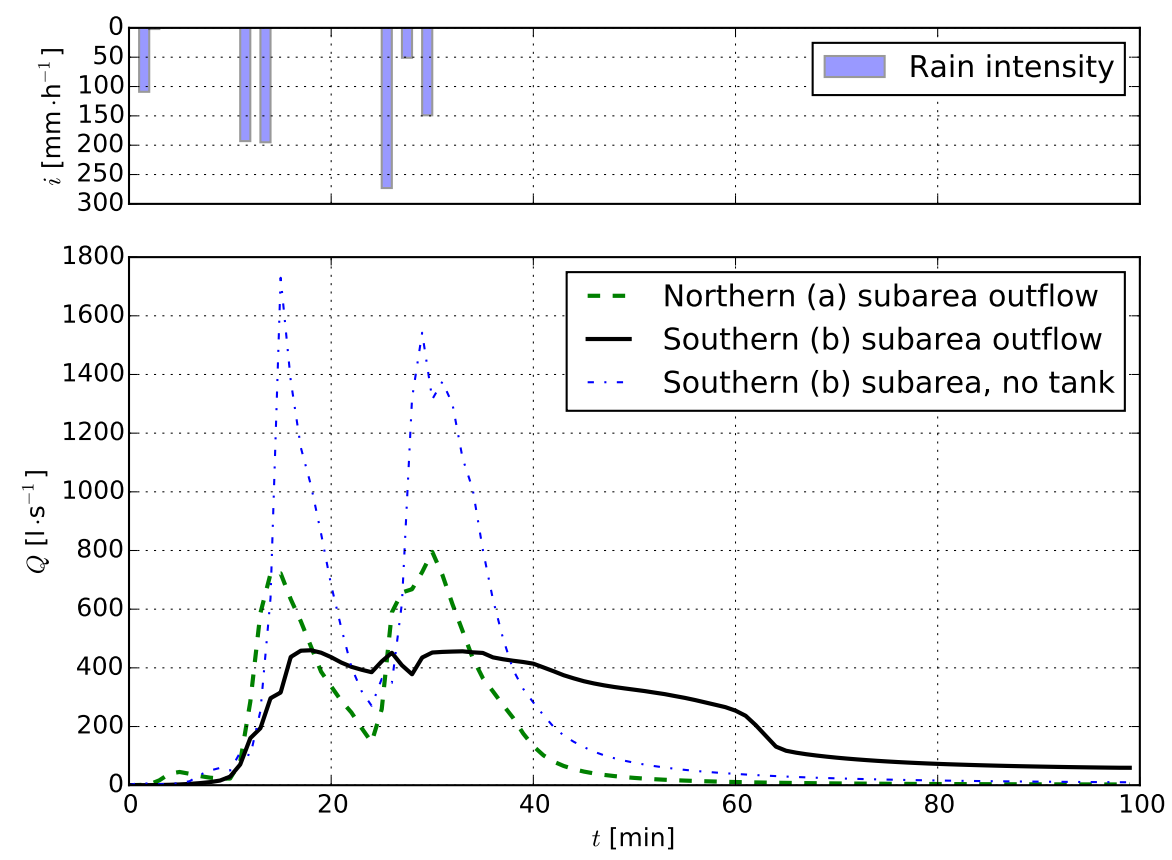

Figure 2: This figure shows SWMM outputs used of both the grassy northern (node (a) in Figure 1) and the urban southern (node (b)) parts of the catchment. We also show the (artificially generated) rain event that was used to produce the outflow. From the plot, we see that the response time $\tau$ of the whole catchment is non-zero. We also see that this short, but intense, rain event triggers the storage tank use (which causes the "step" between 60 and 70 minutes).

\begin{tabular}{c|c|c} 
Auxiliary parameter $\boldsymbol{\theta}^{\prime}$ & Unit & Abbrev. \\
\hline \hline surface reservoir $a$ coefficient & $\mathrm{m}^{\frac{2}{3}}$ & $k_{\text {surf }, a}$ \\
sewer reservoir $b$ coefficient & $\mathrm{m}^{-\frac{1}{3}}$ & $k_{n e t, b}$ \\
surface reservoir $a$ delay & $\mathrm{s}$ & $\tau_{a}$ \\
surface reservoir $a$ depth observation coef. & - & $\mu_{a}$ \\
amplitude of the Gaussian white noise & - & $\sigma$ \\
correlation length & - & $\gamma$ \\
\hline \hline
\end{tabular}

Table 2: List of auxiliary parameters, which cannot be related to SWMM parameters and need to be estimated. These are introduced later in the text. 


\section{Methods}

SWMM is implemented as a system of ordinary differential equations,

$$
\frac{\mathrm{d} \boldsymbol{\Xi}}{\mathrm{d} t}=f(t, p(t), \boldsymbol{\Xi}, \boldsymbol{\theta}),
$$

where $\boldsymbol{\Xi}(t) \in X$ denotes the time-dependent system state, $p(t)$ is the precipitation (in our case a single fixed rain event) and $\boldsymbol{\theta}$ are the parameter factors that we feed into SWMM. The system state is defined as a vector of water storages in the individual building blocks (subcatchments, manholes, discretized tubes, 190 etc.) that make up our SWMM model. For our purpose, the output of SWMM is considered to be a time-series of only few observable components (e.g. discharge at the WWTP or water depths in a few manholes), given by

$$
\mathbf{y}_{i}=\mathbf{g}\left(\boldsymbol{\Xi}\left(t_{i}\right), \boldsymbol{\theta}\right), \quad i=1, \ldots, N_{t},
$$

where $\mathbf{g}$ is a time-independent observation function. The number of observable components (and therefore the dimension of $\mathbf{y}_{\mathbf{i}}$ is later denoted as $N_{x}$ ).

In this section, we build an emulator for (2) and (3) through conditioning a stochastic simplification of (2) and (3) to design data, generated by (2) and (3). The stochastic simplification consists of (i) finding an adequate low-dimensional simplification, $X^{\prime}$, of the state space $X$, (ii) finding an adequate system of linear ODEs, for these simplified state variables, which is inspired from (2) and (3), 200 and (iii) adding noise to the resulting system of ODEs in order to make up for all the simplifications. For our purpose, the design data consists of pairs of time-independent parameter factors $\boldsymbol{\theta}$ and corresponding model outputs (3).

\subsection{Simplification of $S W M M$}

In this subsection, we show how we simplify equations (2) and (3), but we do not give a generic recipe on how to do this. In our framework, any simplification is allowed as long as the simplified equations are linear with respect to the state variables and our method is just one possibility. The imperfections of the simplified model are mitigated by a conditioned Gaussian process, introduced in the next section. The imperfections are due to both the simplification of the state space and the omission of non-linearities. The previously introduced storage tank is an example of such a non-linearity, as can be seen in figure 2 . Bear in mind that the simplified model underlying the emulator needs to be linear with respect to the (reduced) state variables, but not with respect to the SWMM parameters. The better the simplified model manages to capture the 215 non-linear dependence between parameter factors $\boldsymbol{\theta}$ and model outputs (3), the less design data will be needed to correct for the errors.

SWMM components used as a basis of the simplified model. Two essential SWMM components, which are necessary for SWMM to produce a hydrograph are a surface rainfall-runoff model and a channel routing model. In addition, SWMM 
infiltration and evapotranspiration requires solving an implicit equation numerically at each time step (Huber et al., 1988) ${ }^{2}$, which is time consuming and we will therefore use a simplified version of these processes introducing auxiliary parameters (see below).

225 Simplification of the surface-runoff model. In SWMM, a subcatchment $a$ is modelled as a non-linear reservoir with a water height $h_{a}[\mathrm{~m}]$ on its surface as a state variable (Huber et al., 1988). We are interested in the outflow $Q_{a}(t)\left[\mathrm{m}^{3} \cdot \mathrm{s}^{-1}\right]$ during precipitation $p_{a}(t)\left[\mathrm{m} \cdot \mathrm{s}^{-1}\right]$ (each subcatchment can recieve different a amount of precipitation, hence the index a). The continuity equation for the reservoir reads

$$
r_{a} A_{a} \frac{\mathrm{d} h_{a}(t)}{\mathrm{d} t}=r_{a} A_{a} p_{a}\left(t-\tau_{a}\right)-Q_{a}(t),
$$

where $A_{a}\left[\mathrm{~m}^{2}\right]$ is the subcatchment's area multiplied by its imperviousness $r_{a}$ [-] and $\tau_{a}$ is the response time of the subcatchment. The non-linear reservoirs used in SWMM, for the surface runoff, are determined by Manning's equation,

$$
Q_{a}(t)=\frac{w_{a} \sqrt{s_{a}}}{n_{\text {surf }, a}} h_{a}^{\frac{5}{3}}(t)
$$

where $w_{a}[\mathrm{~m}]$ is the width of the subcatchment, $s_{a}[-]$ its slope and $n_{s u r f, a}$ $\left[\mathrm{m}^{-\frac{1}{3}} \cdot \mathrm{s}\right]$ its Manning's roughness coefficient. These equations are already simplified, as they do not include evapotranspiration and infiltration. We have substituted these two processes by the parameter $r_{a}[-]$, which is the fraction of rainfall routed into the sewer network. Furthermore, we have introduced the parameter $\tau_{a}[\mathrm{~s}]$, which represents the response time of the subcatchment. In 240 reality, we never see outflow from a subcatchment immediately after the rain starts and SWMM introduces this effect through wetting processes. We omit these processes in the model simplification and the response time parameter makes up for this. This parameter has to be estimated, which is done later on.

We approximate the non-linear reservoir with a linear one,

$$
Q_{a}(t)=k_{\text {surf }, a} \frac{w_{a} \sqrt{s_{a}}}{n_{\text {surf }, a}} h_{a}(t)=\kappa_{a}\left(\boldsymbol{\theta}, \boldsymbol{\theta}^{\prime}\right) h_{a}(t)
$$

where the linearisation constant $k_{\text {surf,a } a}\left[\mathrm{~m}^{\frac{2}{3}}\right]^{3}$ is estimated (which we do later in the text). Now, the model parameters $w_{a}, n_{\text {surf }, a}$ and $s_{a}$ are fixed for a

\footnotetext{
${ }^{2}$ For the lack of newer documentation of SWMM model mechanisms, we cite the SWMM4 manual even though we are using SWMM5 in this work. This is valid as the principal SWMM components did not change substantially between the two versions.

${ }^{3}$ Contrary to Machac et al. (2015), we retain the scaling of $w_{a}$, in the linear approximation, and use a linearisation constant that is not dimensionless.
} 
subcatchment $a$, but we would like to vary them. The coefficient $\kappa_{a}\left(\boldsymbol{\theta}, \boldsymbol{\theta}^{\prime}\right)$ $\left[\mathrm{m}^{2} \cdot \mathrm{s}^{-1}\right]$ is then seen to depend non-linearly on these SWMM parameters, which are multiplied by the parameter factors $\boldsymbol{\theta}$. Vector $\boldsymbol{\theta}^{\prime}$ contains all the auxiliary parameters of the simplified stochastic model underlying the emulator (see Table $2)$, which need to be estimated through the design data.

Plugging (6) into (4) (and introducing the factor $\theta_{1}$ for $r_{a}$ ) yields the linear differential equation

$$
\frac{\mathrm{d} h_{a}(t)}{\mathrm{d} t}=p_{a}\left(t-\tau_{a}\right)-\frac{\kappa_{a}\left(\boldsymbol{\theta}, \boldsymbol{\theta}^{\prime}\right)}{r_{a} A_{a}} h_{a}(t),
$$

which approximates the behaviour of the SWMM surface-runoff model, for a single subcatchment.

This simplified model, through the vector $\boldsymbol{\theta}$ is connected to some of the SWMM parameter factors, for which we want to build our emulator (Table 1). However, it describes the dynamics of a single subcatchment and the SWMM model contains hundreds of them, each with its own parameters $w, s, n_{\text {surf }}$ and $r$. To retain the speed of the emulator, we cannot represent every subcatchment in the simplified model by a dedicated linear reservoir. In fact, we want to keep 260 the number of linear reservoirs in the simplified model very small $(m \leq 3)$ and we need to aggregate SWMM subcatchments into larger ones. Therefore, all four parameters, $w, s, n_{\text {surf }}$ and $r$, are aggregated as weighted averages, where the area of each subcatchment is the weight. The parameter $A$ in (7) is the sum of the areas of the aggregated subcatchments. This parameter aggregation was done only with the purpose of building a simplified model, which should increase the accuracy of the whole emulator. The remaining surface parameter factors, related to the depression storage, are not included in our simplified model and enter the emulator only through a conditioned Gaussian process, as is explained later.

Further in the text, we call a linear reservoir approximating a surface-runoff model a surface reservoir.

Simplification of the channel routing model. SWMM uses shallow water equations to model channel routing (Huber et al., 1988). Their spatial discretization, necessary for the implementation, yields serially connected reservoirs. In order 275 to find a linear approximation of this part we start with the 1D kinematic wave approximation $^{4}$ of the shallow water equations,

$$
\frac{\partial Q(t, x)}{\partial t}+c \frac{\partial Q(t, x)}{\partial x}=0,
$$

where $c\left[\mathrm{~m} \cdot \mathrm{s}^{-1}\right]$ is the wave celerity. If we now spatially discretize (8), we get

\footnotetext{
${ }^{4}$ This does not restrict us to emulate more complex approximations in SWMM, e.g. the dynamic wave approximation.
} 


$$
\frac{\partial Q(t, x+\Delta x)}{\partial t}+c \frac{Q(t, x+\Delta x)-Q(t, x)}{\Delta x}=0
$$

Rewriting the last equation as

$$
\frac{\Delta x}{c} \frac{\mathrm{d} Q_{x+\Delta x}(t)}{\mathrm{d} t}=Q_{x}(t)-Q_{x+\Delta x}(t)
$$

reveals the similarity to the reservoir continuity equation (4), where instead of the rain input, we have an input from the upstream "node". SWMM also uses this logic (Huber et al., 1988), but with the non-linear reservoir release coefficient according to Manning, as was the case in the surface submodule. For a channel $b$, we set

$$
\frac{c_{b}}{\Delta x_{b}}=\lambda_{b}\left(\boldsymbol{\theta}, \boldsymbol{\theta}^{\prime}\right):=k_{n e t, b} \frac{1}{n_{n e t, b}},
$$

where $\lambda_{b}\left(\boldsymbol{\theta}, \boldsymbol{\theta}^{\prime}\right)\left[\mathrm{s}^{-1}\right]$ is a release coefficient of the linear reservoir, $n_{n e t, b}\left[\mathrm{~m}^{-\frac{1}{3}} \cdot \mathrm{s}\right]$ 285 is the Manning-Strickler coefficient of the channels and $k_{n e t, b}\left[\mathrm{~m}^{-\frac{1}{3}}\right]^{5}$ is another auxiliary parameter. Into this parameter, we have aggregated width and slope of the channel used in Manning's formula, as factors of these parameters are not varied during the emulation ${ }^{6}$. As introduced previously in the surface submodule, $\boldsymbol{\theta}$ is the vector of parameter factors and $\boldsymbol{\theta}^{\prime}$ is the vector which contains auxiliary parameters, in this case $k_{n e t, b}$. If we then use storage $S_{b}\left[\mathrm{~m}^{3}\right]$ of the reservoir as a state variable, we approximate a single node of the SWMM channel routing model with a linear reservoir described by equation

$$
\frac{\mathrm{d} S_{b}(t)}{\mathrm{d} t}=Q_{x-\Delta x, b}(t)-\lambda_{b}\left(\boldsymbol{\theta}, \boldsymbol{\theta}^{\prime}\right) S_{b}(t),
$$

where $x-\Delta x$ denotes the upstream node.

The only connection between a SWMM channel routing module and its 295 linear approximation is then factor $\theta_{7}$ of the Manning-Strickler coefficient of the channels, $n_{n e t, b}\left[\mathrm{~m}^{-\frac{1}{3}} \cdot \mathrm{s}\right]$. Depending on how we divide the channel network, we also aggregate $n_{\text {net }, b}$ parameter as a weighted average, where lengths of channels are used as weights. In our case study, the $n_{n e t, b}$ parameter is the same for the whole network. Further in the text, we call this type of linear reservoir the sewer reservoir.

\footnotetext{
${ }^{5}$ To accurately represent SWMM, in (11), we chose $n_{\text {net }}$ to have power -1 , which leads to this dimension of constant $k_{n e t}$, unlike in Machac et al. (2015), where this constant is dimensionless.

${ }^{6}$ Usually, these parameters are well known from the catchment's topography and the channel geometry, unlike the channel roughness $n_{n e t}$, which needs to be estimated or calibrated.
} 
Simplified model of the whole catchment. For our simplified model, we represent the catchment by a weighted rooted tree with only few vertices (reservoirs). That is, we do not allow for loops in our network and every reservoir has exactly one outflow. Thus, we consider only catchments with one outflow, which is associated with the root of the tree. The leaves of the tree are the surface reservoirs. Each vertex is weighted with its release coefficient, which, depending on whether it is a surface or a sewer reservoir, is given by $\kappa_{a}\left(\boldsymbol{\theta}, \boldsymbol{\theta}^{\prime}\right) /\left(r_{a} A_{a}\right)$ or $\lambda_{a}\left(\boldsymbol{\theta}, \boldsymbol{\theta}^{\prime}\right)$, respectively. We denote the simplified state of our system by the vector $\boldsymbol{\xi} \in X^{\prime}$, whose components are aggregates of the full system's $\boldsymbol{\Xi}$ components. Depending on whether they denote a surface or a sewer reservoir, they are given by the water height $h$ or the storage $S$, respectively. Finally, the simplified dynamics of the catchment is described by the system of coupled linear ODEs

$$
\frac{\mathrm{d} \boldsymbol{\xi}(t)}{\mathrm{d} t}=\mathbf{F}\left(\boldsymbol{\theta}, \boldsymbol{\theta}^{\prime}\right) \boldsymbol{\xi}(t)+\mathbf{P}\left(t, \boldsymbol{\theta}^{\prime}\right)
$$

where matrix $\mathbf{F}\left(\boldsymbol{\theta}, \boldsymbol{\theta}^{\prime}\right)$ is derived from the tree in the obvious manner: matrix element $F_{a b}\left(\boldsymbol{\theta}, \boldsymbol{\theta}^{\prime}\right)$ contains the negative weight of vertex $b$, if $a$ and $b$ are connected by an edge and 0 otherwise. Furthermore, the diagonal elements (which could be represented as loop edges in the tree) contain the weights of the vertices. The precipitation vector $\mathbf{P}\left(t, \boldsymbol{\theta}^{\prime}\right)$ has non-zero elements only at the positions that correspond to surface reservoirs and the input is the same for each of these reservoirs ${ }^{7}$. The input to these reservoirs is delayed by the previously introduced response time $\tau$,

$$
P_{a}\left(t, \boldsymbol{\theta}^{\prime}\right)=p_{a}\left(t-\tau_{a}\right)
$$

To sum up, the simulation model depends on all parameter factors contained in 310 the vector $\boldsymbol{\theta}$ and the simplified one underlying the emulator depends on some of them. The parameter vector $\boldsymbol{\theta}^{\prime}$ is, on the other hand, used only by the simplified model and contains the parameter $\tau_{a}$ and the linearization parameters $k_{n e t, b}$ and $k_{\text {surf,a }}$, where the index $a$ runs over all surface reservoirs and the index $b$ runs over all sewer reservoirs. Furthermore, it contains parameters describing the noise that is added to (13) in the next subsection.

\subsection{Mechanism-based emulator}

The emulator we introduce is based on a system of linear stochastic ordinary differential equations, where the noise term is a Gaussian process of the emulated parameter factors, conditioned on the design data. The design data consist 320 of pairs of simulator inputs (parameter factors) and corresponding simulator outputs (time series as shown in Figure 2). The design data set needs to be generated only once and we then emulate simulator outputs for inputs that are

\footnotetext{
${ }^{7}$ This is usually not true for larger catchments than ours, where we have multiple rain measurements.
} 
not contained in the design data set. The output of the emulator, for a given vector of parameter factors, is a normal distribution, whose mean is the best guess for the corresponding simulator output and whose variance estimates its error. The latter shrinks to zero if the parameter vector approaches one that is part of the design data - the emulator is exact.

Construction of a mechanism-based emulator. In order to build an emulator that is conditioned on $n$ design data sets, we couple $n+1$ replica of (13), where the $(n+1)$-st replica depends on the vectors of parameter factors that we want to emulate. The dynamics of the coupled system is given by equation

$$
\frac{\mathrm{d} \widetilde{\boldsymbol{\xi}}(t)}{\mathrm{d} t}=\widetilde{\mathbf{F}}\left(\widetilde{\boldsymbol{\theta}}, \boldsymbol{\theta}^{\prime}\right) \widetilde{\boldsymbol{\xi}}(t)+\widetilde{\mathbf{P}}\left(t, \boldsymbol{\theta}^{\prime}\right)+\mathbf{C}\left(\widetilde{\boldsymbol{\theta}}, \boldsymbol{\theta}^{\prime}\right) \boldsymbol{\eta}(t), \quad \widetilde{\boldsymbol{\xi}} \in \mathbb{R}^{n+1} \otimes X^{\prime}
$$

We use Greek indices for replica and define the tensors $\widetilde{\mathbf{F}}$ and $\widetilde{\mathbf{P}}$ as

$$
\widetilde{\mathbf{F}}_{\beta}^{\alpha}\left(\widetilde{\boldsymbol{\theta}}, \boldsymbol{\theta}^{\prime}\right)=\delta_{\beta}^{\alpha} \mathbf{F}\left(\boldsymbol{\theta}^{\alpha}, \boldsymbol{\theta}^{\prime}\right) \text { and } \widetilde{\mathbf{P}}^{\alpha}\left(t, \boldsymbol{\theta}^{\prime}\right)=\mathbf{P}\left(t, \boldsymbol{\theta}^{\prime}\right)
$$

where $\delta_{\beta}^{\alpha}$ is the Kronecker delta. The state of the coupled system is $\widetilde{\xi}:=$ $\left(\boldsymbol{\xi}_{1}, \ldots, \boldsymbol{\xi}_{n+1}\right)$ and the vector of parameter factors reads $\widetilde{\boldsymbol{\theta}}:=\left(\boldsymbol{\theta}^{1}, \ldots, \boldsymbol{\theta}^{n+1}\right)$.

The last term in equation (15) is a noise term through which the replica are coupled. The components of $\boldsymbol{\eta}$ are uncorrelated and describe Gaussian white noise. The coupling is defined through

$$
\mathbf{C}\left(\widetilde{\boldsymbol{\theta}}, \boldsymbol{\theta}^{\prime}\right)=\sigma \mathbf{R}\left(\widetilde{\boldsymbol{\theta}}, \boldsymbol{\theta}^{\prime}\right)
$$

where $\sigma$ is the amplitude of the noise and the square of the relative amplitude $\mathbf{R}$ fulfils

$$
\left(\mathbf{R R}^{T}\right)^{\alpha \beta}\left(\widetilde{\boldsymbol{\theta}}, \boldsymbol{\theta}^{\prime}\right)=\exp \left(-\sum_{l}\left(\frac{\theta_{l}^{\alpha}-\theta_{l}^{\beta}}{\gamma_{l}}\right)^{q}\right)
$$

so that the closer to each other the vectors of parameter factors $\boldsymbol{\theta}^{\alpha}$ and $\boldsymbol{\theta}^{\beta}$ are, the stronger the coupling between them is. Together with the noise amplitude $\sigma$, the correlation lengths $\gamma_{l}$ contribute to the auxiliary parameters (replica non-specific) $\boldsymbol{\theta}^{\prime}$ and are estimated later in the text. We can use an arbitrary

335 correlation function instead of (18), as long as its value decreases with greater distance between the vectors of parameter factors. The form that we use is the simplest case of an exponential decay, where, as we found out by trial, the exponent $q$ has very little influence on the result as long as $q$ is an even integer. We therefore choose $q=2$ as is often done with Gaussian process emulation without a dynamic model (O'Hagan, 1992; Gramacy and Lian, 2012).

Finally, we need a linear approximation to the observation equation (3). For simplicity, and despite the aggregation of the state space $X \rightarrow X^{\prime}$, we choose 
the diagonal form

$$
(H[\widetilde{\boldsymbol{\xi}}])_{i a}^{\alpha}= \begin{cases}\mu_{a} \xi_{a}^{\alpha}\left(t_{i}\right) & \text { water depth, } \\ \kappa_{a}\left(\boldsymbol{\theta}^{\alpha}, \boldsymbol{\theta}^{\prime}\right) & \text { discharge of a catchment res. } \\ \lambda_{a}\left(\boldsymbol{\theta}^{\alpha}, \boldsymbol{\theta}^{\prime}\right) & \text { discharge of a sewer res, }\end{cases}
$$

where the particular case depends on the type of the quantity that what we want to observe. The parameter $\mu_{a}$ is a dimensionless auxiliary parameter added to $\boldsymbol{\theta}^{\prime}$ and estimated. The modelled simulator output is then given by (19), i.e.

$$
y_{i a}^{\alpha}=(H[\widetilde{\boldsymbol{\xi}}])_{i a}^{\alpha} .
$$

From eqs. (15) through (20) we derive mean and covariance matrix of the emulator output. As described in detail by Albert (2012) and Machac et al. (2015), they are derived through conditioning the multivariate normal output of the $n+1$ coupled replica to $n$ design data outputs and read as

$$
\begin{aligned}
& \overline{\mathbf{y}}=\mathbf{z}^{n+1}+\Sigma^{n+1, \alpha}\left(\Sigma^{\prime}\right)_{\alpha \beta}^{-1}\left(\mathbf{y}^{\beta}-\mathbf{z}^{\beta}\right), \\
& \bar{\Sigma}=\Sigma^{n+1, n+1}-\Sigma^{n+1, \alpha}\left(\Sigma^{\prime}\right)_{\alpha \beta}^{-1} \Sigma^{\beta, n+1},
\end{aligned}
$$

where $\mathbf{z}$ and $\Sigma$ denote mean and covariance matrix of the unconditioned system, respectively.

Submatrix $\Sigma^{\prime}$, which is associated with the first $n$ replica (design data) of the unconditioned system, needs to be inverted only once during the conditioning phase of the emulation. This is beneficial, since the emulation itself is then very fast. Indeed, in (Albert, 2012), a recursion has been derived that allows calculating $\overline{\mathbf{y}}$ with $\mathcal{O}\left(n N_{t}\right)$ matrix multiplications of order $m$. But it can also be limiting, if the matrix is very large. How to speed-up the inversion is discussed in more detail in the later sections of this article.

Creating design data. The simplified model provides a rough estimate of the SWMM output based on a small number of aggregated parameters and their factors. To pass the information about the simulator that is not contained in the simplified model to the emulator, we need to generate appropriate design data. In order to cover the space of parameter factors equally, we sample a set of 355 parameter factors with the Latin hypercube method (McKay et al., 1979), run the simulator for each of these inputs and save both the outputs and the inputs. The design data are generated with the full SWMM model, which depends on the full range of unaggregated parameters and their factors (which also figure in the simplified models).

360 The design data inputs have to span the same (or larger) range as the emulated inputs, that is

$$
\max _{\alpha}\left(\theta_{l}^{\alpha}\right) \geq 1.5 \text { and } \min _{\alpha}\left(\theta_{l}^{\alpha}\right) \leq 0.5, \quad \forall l .
$$

Outputs of the simulator, for parameter factors generated in this fashion, are shown in Figure 2. 
Estimating auxiliary parameters. With the design data available, we estimate the unknown auxiliary parameters $\boldsymbol{\theta}^{\prime}: \gamma_{l}, \sigma, k_{\text {surf }, a}, k_{n e t, a}, \mu_{a}$ and $\tau_{a}$ (see Table 2 for their meaning) with the maximum likelihood method. We simplify the parameter optimization by using one common correlation length, for all parameters,

$$
\gamma_{l}=\gamma, \forall l
$$

which is justified, because we use the same ranges for all parameters. The log-likelihood function maximized during the optimization is

$$
\ln \mathcal{L}\left(\mathbf{y} \mid \boldsymbol{\theta}^{\prime}\right)=-\frac{1}{2} \ln \left|\Sigma^{\prime}\left(\boldsymbol{\theta}^{\prime}\right)\right|-\frac{1}{2}\left(\mathbf{y}-\mathbf{z}\left(\boldsymbol{\theta}^{\prime}\right)\right)^{T}\left(\Sigma^{\prime}\right)^{-1}\left(\boldsymbol{\theta}^{\prime}\right)\left(\mathbf{y}-\mathbf{z}\left(\boldsymbol{\theta}^{\prime}\right)\right),
$$

where $\mathbf{y}$ is the vector of design data outputs, $\mathbf{z}\left(\boldsymbol{\theta}^{\prime}\right)$ is the Gaussian process mean of the design data calculated from the design data parameters and $\Sigma^{\prime}\left(\boldsymbol{\theta}^{\prime}\right)$ is the covariance matrix of the design data. We then plug-in the resulting $\boldsymbol{\theta}^{\prime}$ into the emulator (Bayarri et al., 2007).

\section{Numerical experiments}

With the construction blocks of the simplified model introduced, we need to combine them to build a simplified model which approximately represents the mechanisms of the emulator. We investigate different simplified model complexities with the dimension of state space up to $m=3$ and the dimension of observation space up to $N_{x}=2$ (the two outputs described in 2.1).

We compare different simplified models by means of the accuracy of the associated emulators. That means selecting a vector of parameter factors (which was not used for conditioning), running both SWMM and the emulator for this set and quantifying the difference between them. To quantify the difference be-

385 tween the outputs at $N_{t}$ time points of the simulator output $\mathbf{y}$ and the emulator $\overline{\mathbf{y}}$ output, generated with the test data at the catchment outlet (or manhole) $x$, we use the root mean square error (RMSE),

$$
\operatorname{RMSE}(\mathbf{y}(x), \overline{\mathbf{y}}(x))=\sqrt{\frac{1}{N_{t}} \sum_{i=1}^{N_{t}}\left(\bar{y}_{i}(x)-y_{i}(x)\right)^{2}} .
$$

\subsection{Simplified models with different complexities}

Because of the computational complexity of our numerical experiments (i.e. producing Figures 6 and 7), where we have to repeatedly do the parameter estimation, and because of the cubic complexity we decided to limit ourselves to a state space not larger than $m=3$. We build all possible simplified models that accommodate this restriction (plus a trivial case with a constant zero prior, 


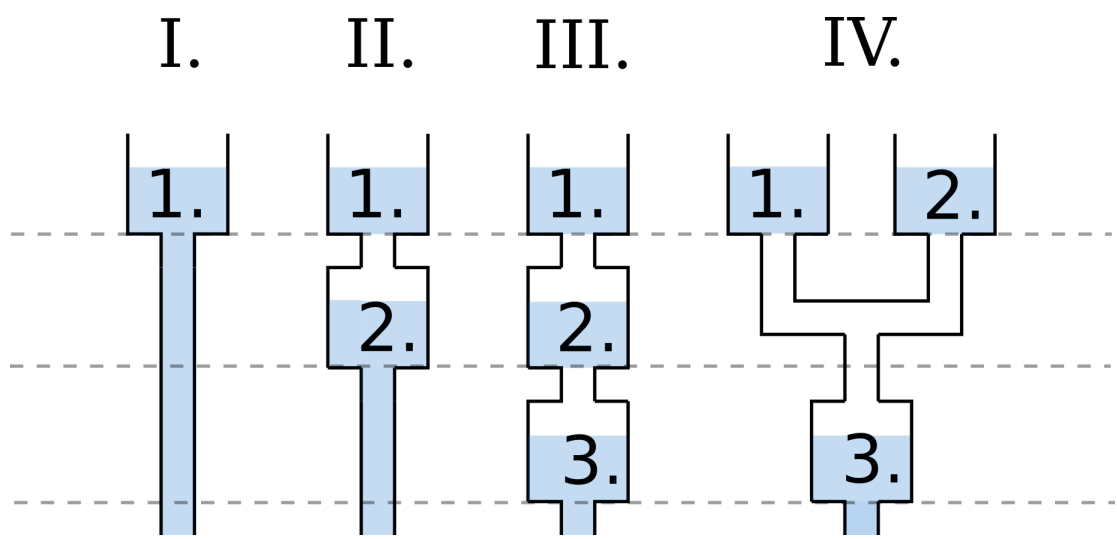

Figure 3: A schematic representation of the cases with different model complexities. The linear reservoirs at the first level always represent the mechanisms of the subcatchment, whereas the following reservoirs represent mechanisms of the channel routing. For cases I-III, we do not take into account different characters of the northern and southern parts of the Adliswil catchment, whereas in the case IV, we do. This is represented by two linear reservoirs on the first level. For the multioutput emulation, we use cases II-IV with the outflow output always being on the lowest level, whereas the water depth output is on the first level for case II, the first or second level for case III and on the first level representation of the southern part of the catchment for case IV.

equivalent to the non-mechanistic emulator) and calculate RMSE for the emulators using these models. For the two-dimensional output emulator, the RMSE is calculated separately for each output. An overview of the simplified models is shown in Figure 3. Only in case IV, we exploit the division of the catchment into two parts as seen in Figure 1.

Case I. The simplest non-trivial linear model consists of a single linear reservoir, which approximates only the surface-runoff model. We expect this to be the least accurate case, because we do not approximate the channel routing model and therefore do not exploit our knowledge of the parameter $n_{n e t}$. The simplified model is expressed as

$$
F^{\alpha}=-\frac{\kappa\left(\boldsymbol{\theta}^{\alpha}, \boldsymbol{\theta}^{\prime}\right)}{r^{\alpha} A}, \quad P=p(t-\tau) .
$$

Since the state space has dimension $m=1$, we model only a single output ${ }^{8}$ and we choose this to be the outflow. The observation operator (19) is then

$$
(H[\widetilde{\boldsymbol{\xi}}])_{i}^{\alpha}=\kappa\left(\boldsymbol{\theta}^{\alpha}, \boldsymbol{\theta}^{\prime}\right) \xi^{\alpha}\left(t_{i}\right) .
$$

\footnotetext{
${ }^{8}$ We can still model more outputs. However, these will all be projected from a single reservoir, which leads to inaccuracies for all the outputs.
} 
Case II. For the second case, we add a sewer reservoir representing the sewer network. The simplified model is then expressed as

$$
\mathbf{F}^{\alpha}=\left(\begin{array}{cc}
-\frac{\kappa_{1}\left(\boldsymbol{\theta}^{\alpha}, \boldsymbol{\theta}^{\prime}\right)}{r_{1}^{\alpha} A_{1}} & 0 \\
\frac{\kappa_{1}\left(\boldsymbol{\theta}^{\alpha}, \boldsymbol{\theta}^{\prime}\right)}{r_{1}^{\alpha} A_{1}} & -\lambda_{2}\left(\boldsymbol{\theta}^{\alpha}, \boldsymbol{\theta}^{\prime}\right)
\end{array}\right), \quad \mathbf{P}=\left(\begin{array}{c}
p\left(t-\tau_{1}\right) \\
0
\end{array}\right)
$$

with the observation operator for the single (outflow) output being

$$
(H[\widetilde{\boldsymbol{\xi}}])_{i 2}^{\alpha}=\lambda_{2}\left(\boldsymbol{\theta}^{\alpha}, \boldsymbol{\theta}^{\prime}\right) \xi_{2}^{\alpha}\left(t_{i}\right) .
$$

Since $m=2$, we can add an additional output on the remaining surface linear reservoir. Since we are interested in the water depth, we have

$$
(H[\widetilde{\boldsymbol{\xi}}])_{i 1}^{\alpha}=\mu_{1} \xi_{1}^{\alpha}\left(t_{i}\right) .
$$

Case III. This case is similar to the previous one, with an extra sewer reservoir. The simplified model is expressed as

$$
\mathbf{F}^{\alpha}=\left(\begin{array}{ccc}
-\frac{\kappa_{1}\left(\boldsymbol{\theta}^{\alpha}, \boldsymbol{\theta}^{\prime}\right)}{r_{1}^{\alpha} A_{1}} & 0 & 0 \\
\frac{\kappa_{1}\left(\boldsymbol{\theta}^{\alpha}, \boldsymbol{\theta}^{\prime}\right)}{r_{1}^{\alpha} A_{1}} & -\lambda_{2}\left(\boldsymbol{\theta}^{\alpha}, \boldsymbol{\theta}^{\prime}\right) & 0 \\
0 & \lambda_{2}\left(\boldsymbol{\theta}^{\alpha}, \boldsymbol{\theta}^{\prime}\right) & -\lambda_{3}\left(\boldsymbol{\theta}^{\alpha}, \boldsymbol{\theta}^{\prime}\right)
\end{array}\right), \quad \mathbf{P}=\left(\begin{array}{c}
p\left(t-\tau_{1}\right) \\
0 \\
0
\end{array}\right)
$$

As for the observation operator, the outflow is again calculated as

$$
(H[\widetilde{\boldsymbol{\xi}}])_{i 3}^{\alpha}=\lambda_{3}\left(\boldsymbol{\theta}^{\alpha}, \boldsymbol{\theta}^{\prime}\right) \xi_{3}^{\alpha}\left(t_{i}\right) .
$$

For the water depth, we can choose either

$$
(H[\widetilde{\boldsymbol{\xi}}])_{i 1}^{\alpha}=\mu_{1} \xi_{1}^{\alpha}\left(t_{i}\right) \text { or }(H[\widetilde{\boldsymbol{\xi}}])_{i 2}^{\alpha}=\mu_{2} \xi_{2}^{\alpha}\left(t_{i}\right)
$$

and we compare the accuracy of both of these options.

Case IV. The final case takes into account the structure of the catchment by using 2 surface reservoirs with different parameters. We expect this case to be the most accurate. The outflow is projected as

$$
(H[\widetilde{\boldsymbol{\xi}}])_{i 3}^{\alpha}=\lambda_{3}\left(\boldsymbol{\theta}^{\alpha}, \boldsymbol{\theta}^{\prime}\right) \xi_{3}^{\alpha}\left(t_{i}\right) .
$$

${ }_{415}$ The simplified model has the form 


$$
\mathbf{F}^{\alpha}=\left(\begin{array}{ccc}
-\frac{\kappa_{1}\left(\boldsymbol{\theta}^{\alpha}, \boldsymbol{\theta}^{\prime}\right)}{r_{1}^{\alpha} A_{1}} & 0 & 0 \\
0 & -\frac{\kappa_{2}\left(\boldsymbol{\theta}^{\alpha}, \boldsymbol{\theta}^{\prime}\right)}{r_{2}^{\alpha} A_{2}} & 0 \\
\frac{\kappa_{1}\left(\boldsymbol{\theta}^{\alpha}, \boldsymbol{\theta}^{\prime}\right)}{r_{1}^{\alpha} A_{1}} & \frac{\left.\kappa_{2} \boldsymbol{\theta}^{\alpha}, \boldsymbol{\theta}^{\prime}\right)}{r_{2}^{\alpha} A_{2}} & -\lambda_{3}\left(\boldsymbol{\theta}^{\alpha}, \boldsymbol{\theta}^{\prime}\right)
\end{array}\right), \quad \mathbf{P}=\left(\begin{array}{c}
p\left(t-\tau_{1}\right) \\
p\left(t-\tau_{2}\right) \\
0
\end{array}\right)
$$

and for the water depth, we can choose either of the two surface reservoirs (1 or 2) with observation

$$
(H[\widetilde{\boldsymbol{\xi}}])_{i 1}^{\alpha}=\mu_{1} \xi_{1}^{\alpha}\left(t_{i}\right)
$$

and both choices lead to the same result.

\subsection{Accuracy of the mechanism-based emulator with different simplified models}

We determine and compare the accuracies of the emulators based on the simplified models described in the previous subsection. For each of these emulators, we calculate the RMSE for 4 to 40 design data sets. For each emulator and each $n$, we calculate the RMSE 100 times (each time we run the emulator with different design data sets) for 1000 test data sets (each time with the same 1000 test data sets) and use the mean RMSE of these 100 runs as our final RMSE value.

At each of the 100 calculations, we randomly sample $n$ design data sets from a 1000 design data sets, generated beforehand. After each sampling, we run our estimation algorithm, simulated annealing by Goffe et al. (1992), to maximize

${ }_{430}$ (25) and find the unknown auxiliary parameters. The computational cost of this step is the reason behind running the simulator/emulator for a shorter time-span. However, this is an issue only for this numerical experiment, where the estimation is repeated 100 times for each $n$.

\subsection{Time gain of a mechanism based emulator with respect to $S W M M$}

We aim at quantifying the time gain of the emulator of SWMM compared to SWMM itself. Therefore, we average both the simulation and the emulation time, the latter without the conditioning phase, over 1000 test sets of parameter factors.

As for the conditioning, the inversion of matrix $\Sigma^{\prime}$ in (21), which is of theoretical complexity $\mathcal{O}\left(\left(N_{t} n m\right)^{3}\right)$, can be reformulated. The matrix inverse only appears as a left multiplier to either a vector $\left(\mathbf{y}^{\beta}-\mathbf{z}^{\beta}\right)$ or a matrix $\Sigma^{\beta, n+1}$. The result of this multiplication can then be obtained as a solution of a system of linear equations with $\Sigma^{\prime}$ being the system matrix and the aforementioned vector $\left(\mathbf{y}^{\beta}-\mathbf{z}^{\beta}\right)$ or matrix $\Sigma^{\beta, n+1}$ being the right hand side. Although solving 445 a system of linear equations is also of theoretical complexity $\mathcal{O}\left(\left(N_{t} n m\right)^{3}\right)$, it is faster than the full matrix inverse. We can further benefit from the symmetry of the covariance matrix $\Sigma^{\prime}$, which allows for a faster solution of a linear system by using routines exploiting this property, such as SSYTRF from the LAPACK 
library (Anderson et al., 1999), which is used in our implementation. Another potential improvement is using GPU parallelization to solve the linear system, such as the method proposed by Legendre et al. (2013), which yields an up to an order of magnitude faster algorithm than those from LAPACK, depending on the hardware used.

For the time comparison with SWMM, we use two implementations of the 455 mechanism-based emulator. One uses Kalman filtering (Reichert et al., 2011) for conditioning the prior to the design data and the second one uses an alternative approach (Albert, 2012).

\section{Results and their interpretation}

\subsection{Emulator accuracy}

Even with the added mechanisms, an emulator is only as good as the design data that it uses. If vector of parameter factors that we emulate for lies far away from the design data, we might expect the that the influence of a good simplified model increases. We therefore use the Euclidean distance of the emulated vector of parameter factors to the closest design vector, defined as

$$
l=l\left(\boldsymbol{\theta}^{n+1}, \widetilde{\boldsymbol{\theta}}\right)=\min _{\alpha \in\{1, \ldots, n\}}\left\|\boldsymbol{\theta}^{\alpha}-\boldsymbol{\theta}^{n+1}\right\|,
$$

${ }_{465}$ to pick a test vector, which lies the furthest away from any design data set. Out of a sample of 1000 test vectors, we have found this to be one with $l=1.35$ and in Figure 4, we plotted the emulated output. We have used a single output emulator, which was conditioned with 40 design data set. There, we see the accuracy improvement between cases I and IV. We therefore benefit from a better simplified model, even if the simplified model itself is still far off the SWMM simulation. This is the main feature of a mechanism-based emulator in comparison to other emulators. In Figure 5, we plotted the output of the simulator for a two-dimensional output case III with a typical distance from the design data, $l=0.9$. Both Figures contain outputs of simplified models used for 475 the emulation and the emulator outputs with their $95 \%$ confidence intervals.

In Figure 6, we see the root mean square errors (26) of the single output emulators. We also added the case with the prior mean of the emulator $\mathbf{z}=0$ (by setting $\mathbf{F}=0$ ), where the emulator includes no information about SWMM's mechanisms other than what it gets from the design data. These results support what was concluded by Machac et al. (2015) - the emulator accuracy increases a lot already with the crudest simplified model as opposed to no model. Increasing the complexity further by linking more simulator parameter factors to the simplified model, which is the difference between cases I and II, has also a large impact on the emulator accuracy. By enlarging the state space with485 out linking additional parameters (difference between II and III), we still gain little accuracy, but it does not justify the increased computational complexity. The change from serial linear reservoirs (III) to parallel linear reservoirs (IV.) 


\begin{tabular}{|c|c|c|c|c|}
\hline Lin. mod. & I. & II. & III. & IV. \\
\hline RMSE, outflow $\left[\mathbf{l} \cdot \mathbf{s}^{-1}\right]$ & 256.75 & 205.74 & 201.34 & 196.85 \\
\hline
\end{tabular}

Table 3: RMSE for different simplified models only (without using the emulator) for singleoutput case. This table serves for comparison with the emulation error shown in Figure 6. The actual simplified model outputs for cases I and IV are shown in Figure 4.

\begin{tabular}{|c|c|c|c|c|}
\hline Lin. mod. & II. & III., $2^{\text {nd }}$ res. & III., $1^{\text {st }}$ res. & IV. \\
\hline RMSE, manhole [m] & 0.47 & 0.57 & 0.43 & 0.48 \\
\hline RMSE, outflow $\left[\mathbf{l} \cdot \mathbf{s}^{-1}\right.$ ] & 206.46 & 202.19 & 201.01 & 196.46 \\
\hline
\end{tabular}

Table 4: RMSE for different simplified models only (without using the emulator) for singleoutput case. This table serves for comparison with the emulation error shown in Figure 7. The reservoir number identifies the point in the state space, from where we read the water depth in the manhole. The outflow is always read from the last reservoir. The actual simplified model output for case III is shown in Figure 5

again provides only a slight improvement. However, since the state space in these cases is equally large, $m=3$, this change does not increase the computational complexity and has therefore only benefits. A different view on the accuracy improvement is shown in Figure 8, where we see a significant accuracy improvement, for test sets that are far from the design data.

In the multi-output emulator, it is important how we connect the outputs of interest with the state space components of the system through the observation 495 operator $H$, as we see in Figure 7 . The best results are again achieved if we take into account the model structure (IV). However, there is a significant difference in where we place the second output for the case III. If we place the depth output on the second reservoir, then during the parameter estimation a compromise is found, that fits neither the outflow nor the depth outputs very well. If we place the depth output on the first reservoir, then we have one reservoir in between the outflow and the depth outputs, which allows for a better fit of both, hence the result of the emulator improves.

\subsection{Emulator computational complexity and time gain}

The results for this paper were generated with the emulator introduced by Albert (2012). One emulation time-step in this implementation is of theoretical complexity $\mathcal{O}\left(n m^{3}\right)$ as opposed to the version of the emulator with Kalman filtering by Reichert et al. (2011), where one step is of complexity $\mathcal{O}\left((\mathrm{nm})^{3}\right)$. As we see in Figure 9 (bottom), which shows the time measurements of our implementation (on a $\log$ scale), the theoretical expectations are fulfilled with respect to the amount of design data $n$.

The trade-off for the better emulation performance of the emulator by Albert (2012) is the slower conditioning (Figure 9, top), which requires solving a system of $N_{t} n m$ equations and is a heavily memory dependent problem. The emulator implementation by Reichert et al. (2011) does not have this problem

when conditioning, however this is quickly offset during the emulation itself, 

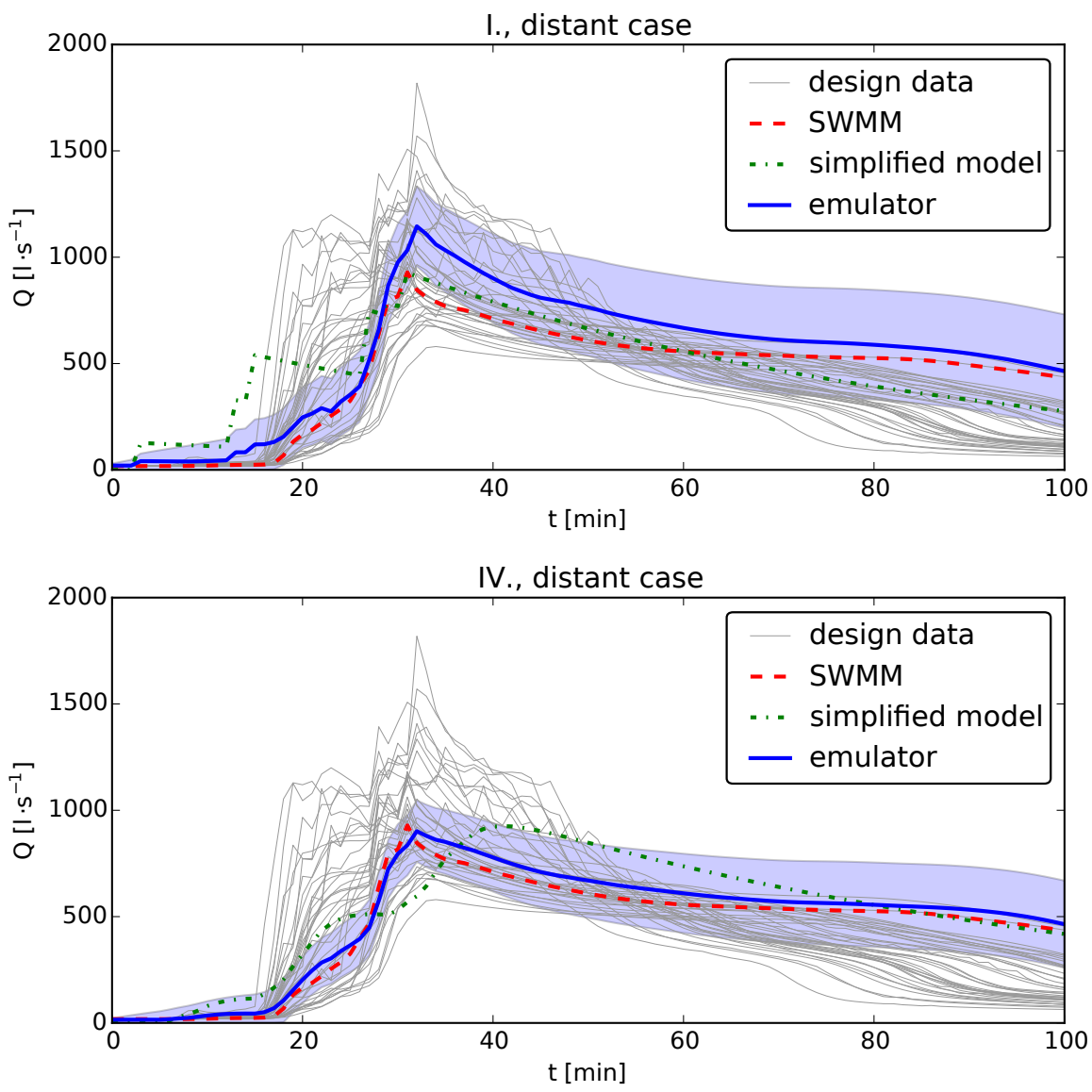

Figure 4: Comparison of two emulator outputs with $95 \%$ confidence interval shown in light blue. The top and bottom plots depict single output cases I and IV, respectively. The amount of design data used is $n=40$. Out of the sample of 1000 test runs, we have picked the test set, which is furthest from the design data $(l=1.35)$ with accuracies RMSE $=137.45$ for the top plot $\mathrm{l} \cdot \mathrm{s}^{-1}$ and RMSE $=63.45 \mathrm{l} \cdot \mathrm{s}^{-1}$ for the bottom one. Additionaly, we show outputs of the corresponding simplified models. 

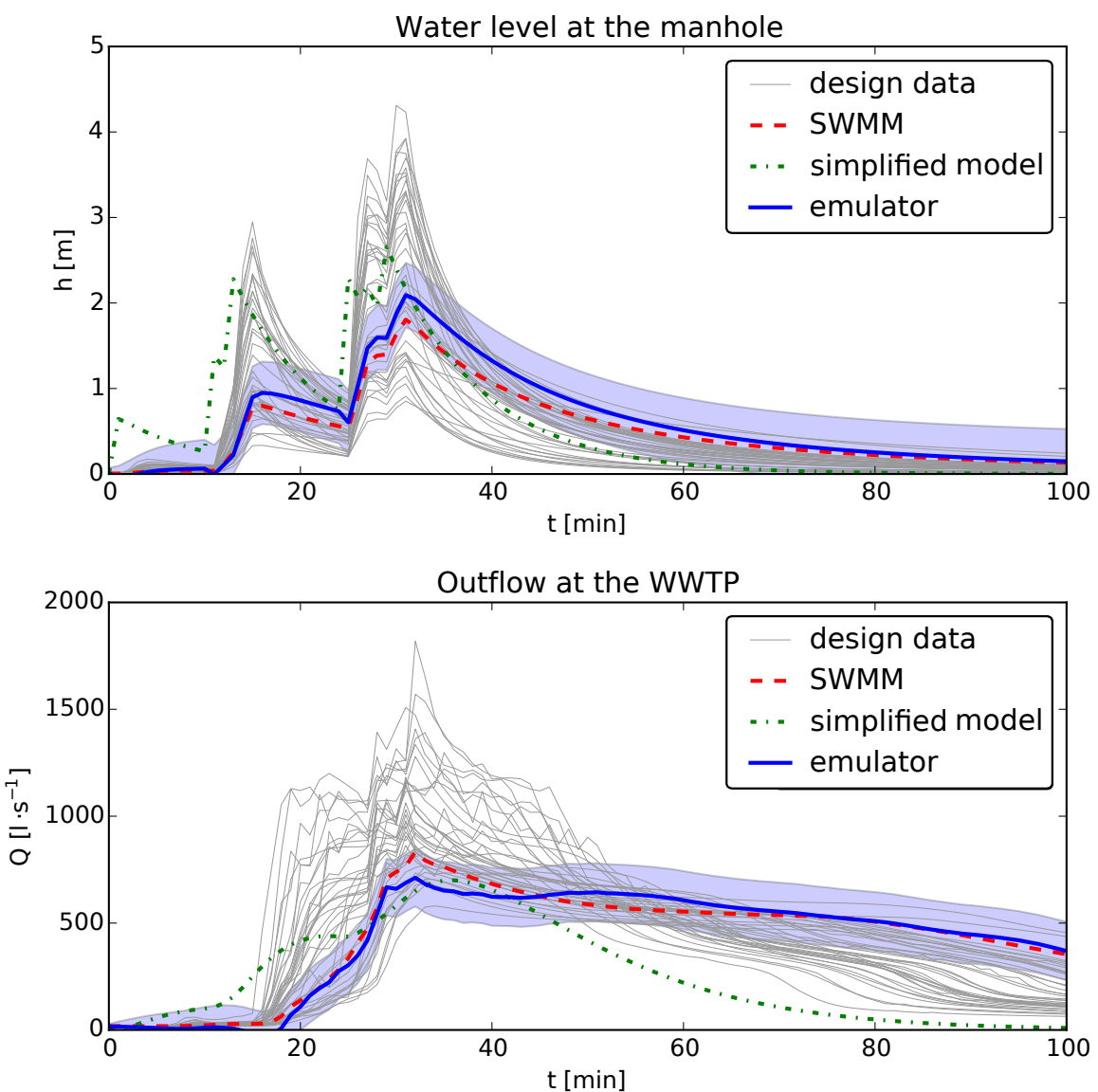

Figure 5: Outputs of a two-dimensional output emulator (case III) with $95 \%$ confidence interval shown in light blue. The top and bottom plots depict the water level at the manhole and the outflow at the WWTP, respectively. We show a typical case with distance from the design data $l=0.9$ and $\mathrm{RMSE}=0.15 \mathrm{~m}$ resp. $\mathrm{RMSE}=58.34 \mathrm{l} \cdot \mathrm{s}^{-1}$ 


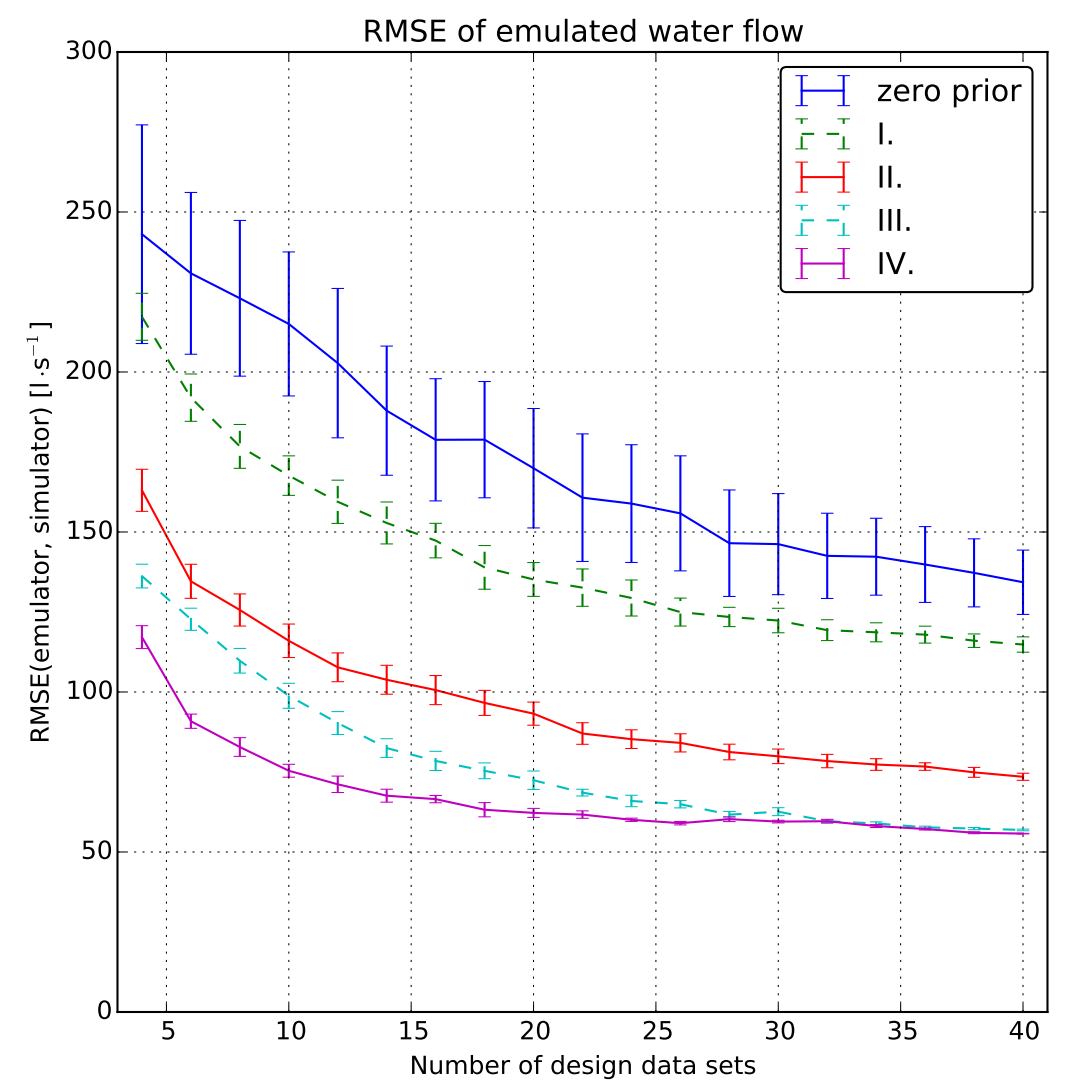

Figure 6: Root mean square error (26) for the single-output emulator of the outflow $Q$ at catchment outlet constructed with various simplified models. The case with zero prior is the least accurate. Case I, which does not include any information about the sewer network in the simplified model, is significantly more accurate than the case with zero prior. All the other cases, which include this information are significantly more accurate with case IV being the most accurate. 

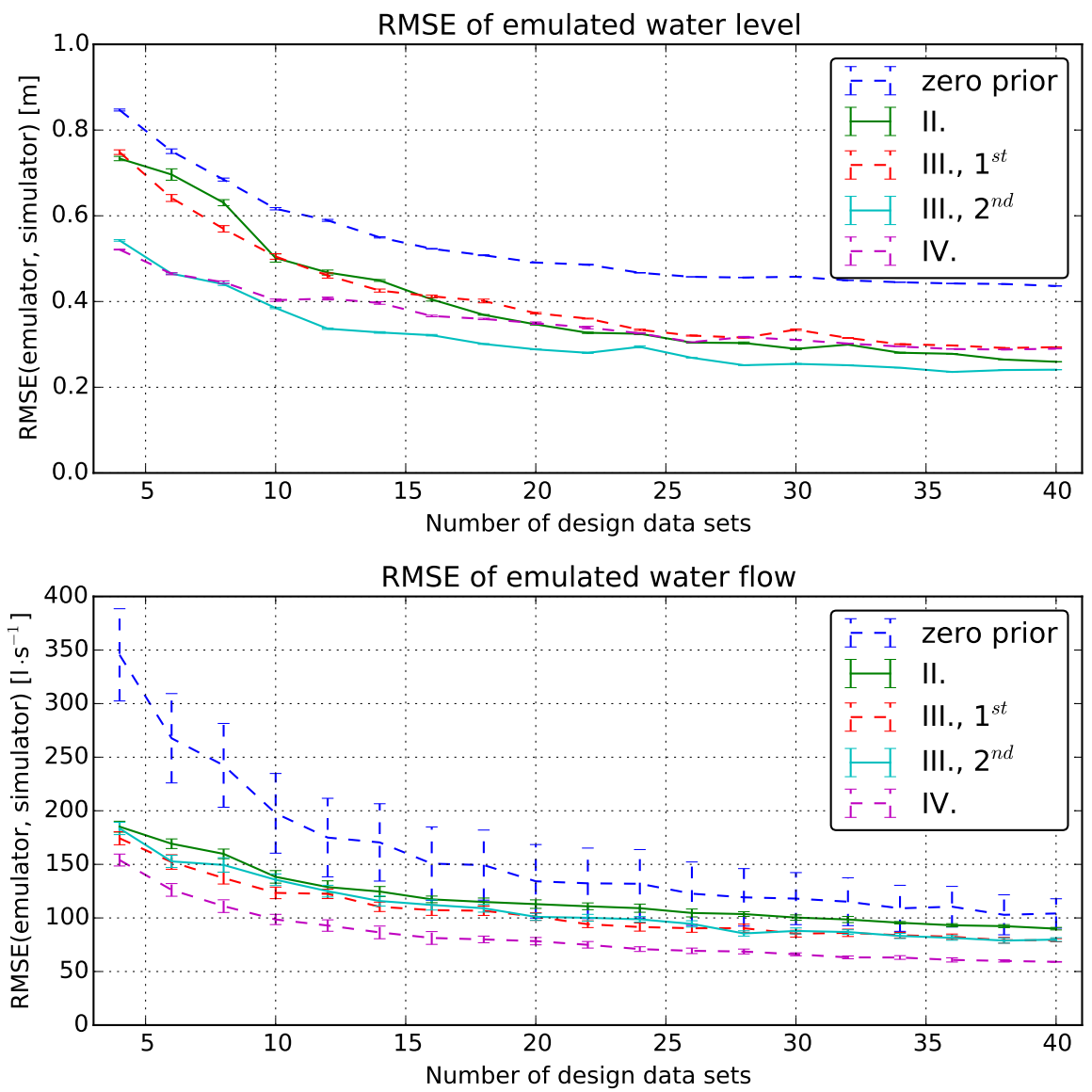

Figure 7: Root mean square error for the multi-output emulator constructed with various simplified models. For the outflow, the situation is similar to the single output-emulator. However, for the water depth, measured upstream of the WWTP inflow, the complexity of the underlying simplified model brings little benefit. 

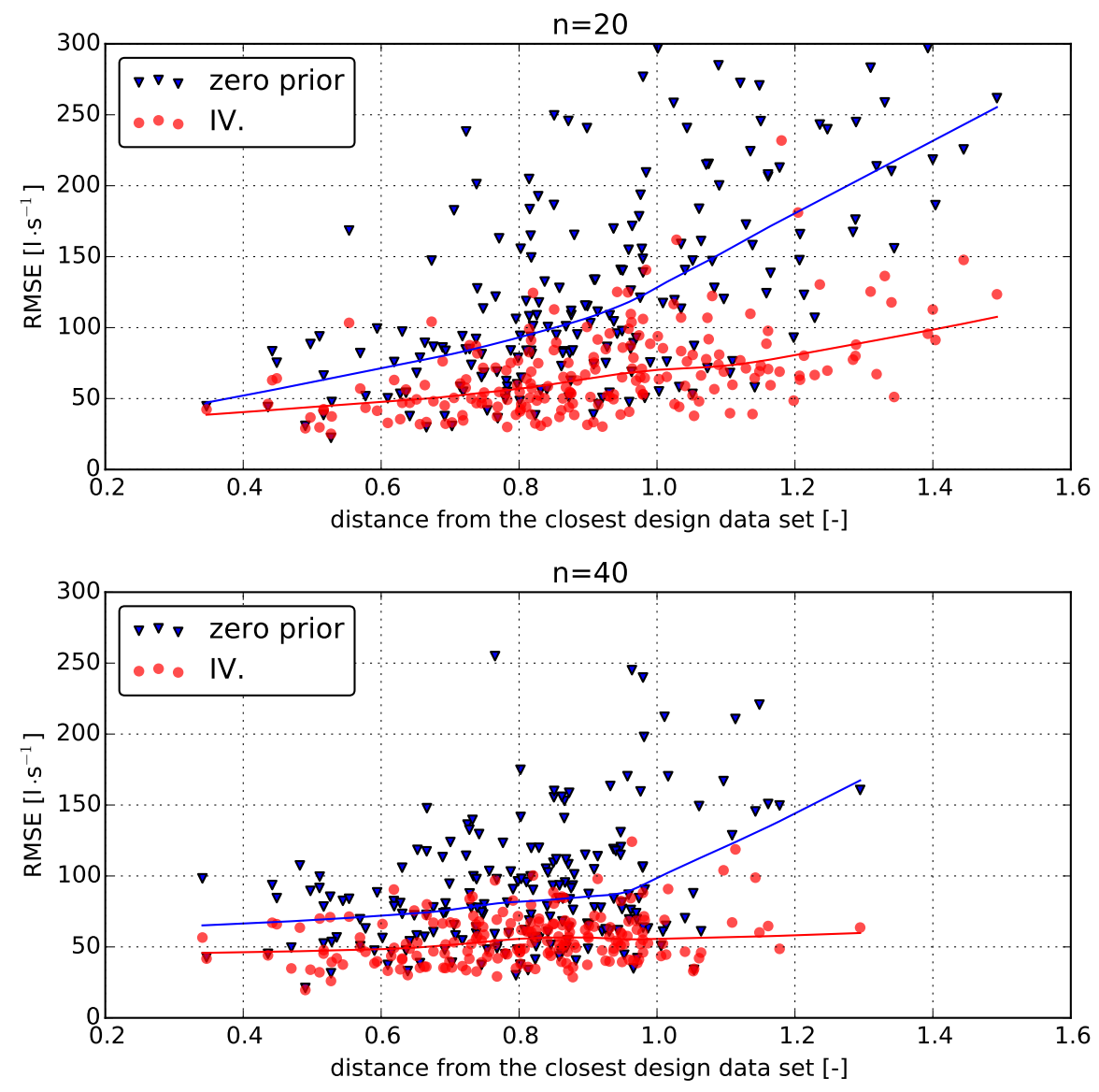

Figure 8: Comparison of RMSE of a single output emulator with zero prior and the case IV for 20 and 40 design data sets. On the horizontal axis, we show the Euclidean distance of a test set from the closest design data set. The benefit of the more complex simplified model is most pronounced for the test sets far from design data. We have used the regression model LOESS (solid lines) to better illustrate this advantage. 
should the number of design data sets rise. Which of the two emulators to use is problem dependent and we should always consider both options.

\section{Practical applications}

Our motivation to construct this type of emulator (emulation with respect to 520 parameters) is the calibration of SWMM models. This means first constructing an emulator of a SWMM model, as described in this article. Then, we use the emulator in our calibration algorithm instead of SWMM. The calibration can be done with the maximum likelihood method, for example, combined with a suitable optimization technique, such as simulated annealing. Once the algorithm ${ }_{525}$ converges, we plug the resulting parameter vector into SWMM and validate that it gives the same output as the emulator. Our preliminary results show that this technique can successfully be used for the calibration of the Adliswil model, with time-series consisting of up to 1500 time-points, with the same results as calibration with the full model, while reducing the calibration time from weeks to hours.

Although the SWMM output can be evaluated at as many sites (nodes, outflows, etc.) as we wish for no extra computational cost, adding many extra outputs slows down the emulator due to its $\mathcal{O}\left(\mathrm{m}^{3}\right)$ scaling with the number of state variables. However, this is not a problem for the calibration, as the number

535 of sites where we conduct measurements is limited. If one needs to use orders of magnitude more outputs, we recommend considering the approach used by Spiller et al. (2014), where a simple emulator is constructed for each output of the simulator.

Another common problem, where emulators are used, is model input uncertainty propagation (Dalbey et al., 2008). If we have model parameters with certain distributions and we want to calculate the distributions of model outputs, only very simple models allow us to do this analytically. Monte Carlo techniques allow us to estimate the output distribution also for complex models, albeit usually with thousands of model runs. These runs can be then substituted

${ }_{545}$ by an emulator. Sensitivity analysis of a model is another problem, where we can deploy emulators.

A typical use case for emulation in urban water drainage is real time control (Schütze et al., 2004) upon which we plan to focus in our future work. After receiving data from sensors distributed in the catchment (flow meters, rain gauges etc.), we want to adjust the actuators of the system so that the strain on the system itself and the environment is minimal. Optimal settings of the actuators can be inferred by model optimization. This optimization, however, can be too slow to be carried out in real-time. Emulation might then make this process faster.

\section{Conclusions}

Our mechanism-based emulator combines information about the structure of the simulator with design data produced by the simulator to construct the 

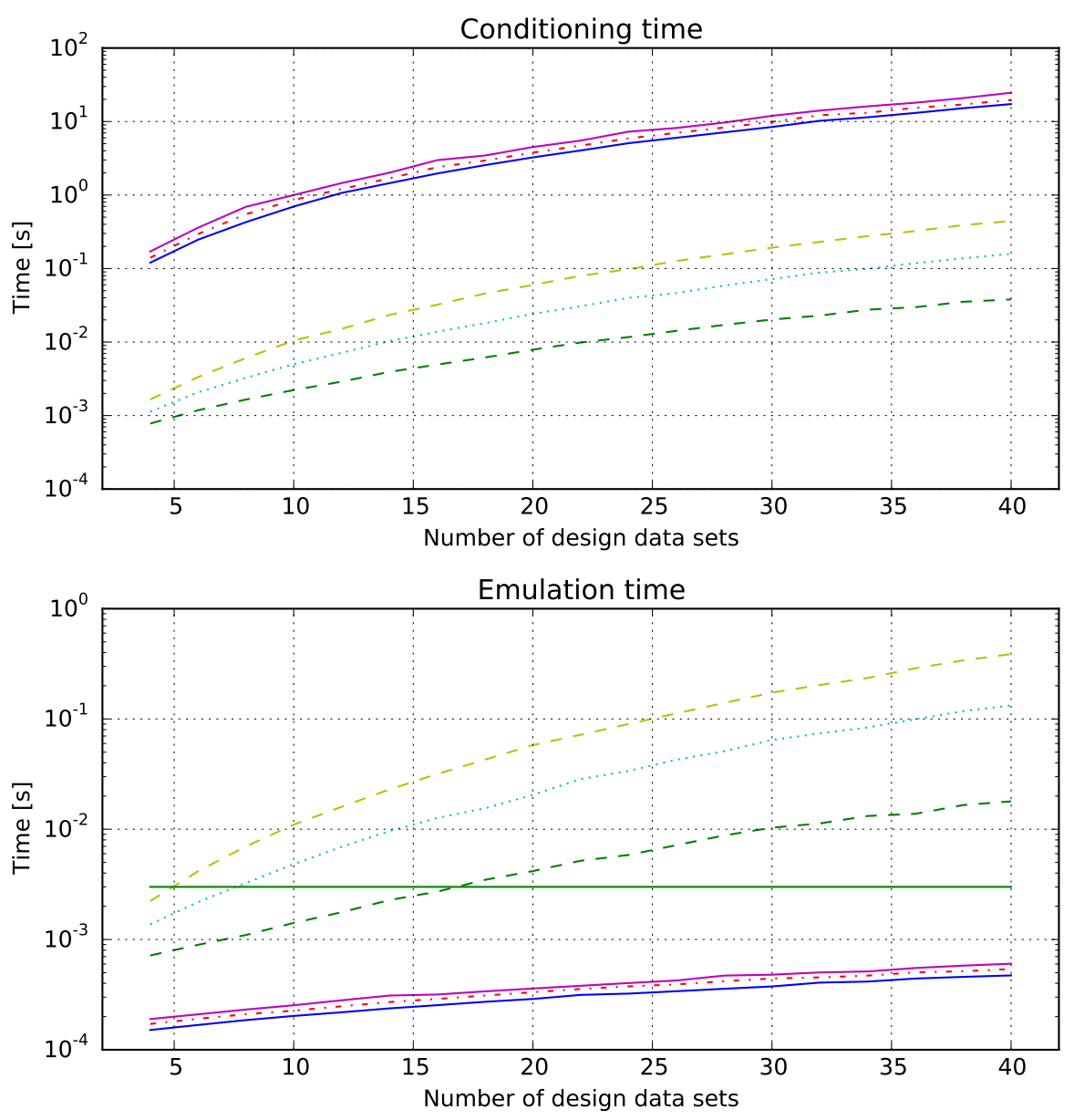

\begin{tabular}{llll}
- & non-Kalman, $\mathrm{m}=1$ & $\cdots$ & Kalman, $\mathrm{m}=2$ \\
$\cdots$ & non-Kalman, $\mathrm{m}=2$ & -- & Kalman, $\mathrm{m}=3$ \\
- & non-Kalman, $\mathrm{m}=3$ & - & $1 / 1000$ SWMM runtime \\
-- & Kalman, $\mathrm{m}=1$ & & \\
\hline
\end{tabular}

Figure 9: Comparison of elapsed time of two different implementations of mechanism-based emulator, by Reichert et al. (2011) (with Kalman filtering) and by Albert (2012) (without Kalman filtering). The top plot shows the time necessary for conditioning, design data generation not included. We condition the emulator only once and this time should therefore not be used to benchmark the emulator. The second plot, on the other hand, provides a good benchmark, as we see emulation times for a single test set. For comparison with the original simulator, which takes approximately 3 seconds, we show a $1 / 1000$ of this time. Both plots use $\log$ scale. 
emulator. The mechanistic model is considered by a simplified, linear, stochastic model on which the emulator is based. As the behavior of this simplified model is corrected by conditioning it to the design data, emulators can be constructed based on different simplified models. The better this simplified model already approximates the behavior of the simulator (as a function of parameters and/or inputs), the less corrections are required by the conditioning process and we can expect that less design data may be required to achieve a given emulation

565 accuracy. On the other hand, more complex simplified models may be more demanding to evaluate during conditioning and emulation. To investigate this behavior in our case study, we used five approximating models of increasing complexity and compared the emulation accuracy as a function of the size of the design data set. Taking into account more of the structure of the catchment leads to more accurate emulation for the same size of the design data set at the expense of computational complexity. The final choice of the approximating simplified model will thus be a case-specific trade-off between improving the mechanistic description or the size of the design data set. This study confirms earlier results, that a relatively crude approximating model may be sufficient 575 to provide a significant gain in accuracy. However, in this case, we still get considerable improvements beyond the simplest model.

As our emulation times are much shorter than those of the original simulator (see Fig. 9), we can conclude that emulation may enable us to reduce the constraints of long computation times that we face for tasks that require many simulation runs in urban drainage modelling. However, our analyses were based on very small design data sets. As is seen in Figs. 6 and 7, the further increase in accuracy, which may be required to use the emulator for parameter estimation as well as to use it for higher dimensions of the parameter space (e.g. to emulate for different input), will need a much larger design data set. This will slow down the emulator unless the efficiency of its numerical implementation is improved.

Further research is thus needed to speed-up the emulator and to make it available for the emulation of input (rainfall time series) in addition to the emulation of model parameters for given input. This would make it available 590 for real-time control of sewer systems, where we need fast predictions of the response of the sewer system to short-term precipitation forecasts.

\section{Acknowledgements}

This work is funded by the Swiss National Science Foundation, grants no. CR22I2 135551 and CR22I2 152824, in scope of the project "Using Commercial ${ }_{595}$ Microwave Links and Computer Model Emulation to Reduce Uncertainties in Urban Drainage Simulations" (COMCORDE). The authors would also like to thank Lorenzo Garbani Marcantini for valuable comments to the manuscript. 


\section{References}

Albert, C., 2012. A mechanistic dynamic emulator. Nonlinear Analysis: Real

600

Anderson, E., Bai, Z., Bischof, C., Blackford, S., Demmel, J., Dongarra, J., Du Croz, J., Greenbaum, A., Hammarling, S., McKenney, A., Sorensen, D., 1999. LAPACK Users' Guide, 3rd Edition. Society for Industrial and Applied Mathematics, Philadelphia, PA.

605

Asher, M., Croke, B., Jakeman, A., Peeters, L., 2015. A review of surrogate models and their application to groundwater modeling. Water Resources Research.

Axworthy, D. H., Karney, B. W., 1999. Modeling surface and subsurface runoff in a forested watershed. Journal of Hydrologic Engineering 4 (2), 165-173.

Bayarri, M. J., Berger, J. O., Cafeo, J., Garcia-Donato, G., Liu, F., Palomo, J., Parthasarathy, R. J., Paulo, R., Sacks, J., Walsh, D., 2007. Computer model validation with functional output. Annals of Statistics 35 (5), 1874-1906.

Bellman, R., 1956. Dynamic programming and lagrange multipliers. Proceedings of the National Academy of Sciences of the United States of America 42 (10), 767 .

Bhattacharya, S., 2007. A simulation approach to Bayesian emulation of complex dynamic computer models. Bayesian analysis 2 (4), 783-816.

Castelletti, A., Galelli, S., Ratto, M., Soncini-Sessa, R., Young, P., 2012. A general framework for dynamic emulation modelling in environmental problems. Environmental Modelling \& Software 34 (0), 5 - 18 .

Conti, S., Gosling, J. P., Oakley, J. E., O'Hagan, A., Jun. 2009. Gaussian process emulation of dynamic computer codes. Biometrika 96 (3), 663-676.

Cressie, N., 1990. The origins of kriging. Mathematical geology 22 (3), 239-252.

Dalbey, K., Patra, A., Pitman, E., Bursik, M., Sheridan, M., 2008. Input uncertainty propagation methods and hazard mapping of geophysical mass flows. Journal of Geophysical Research: Solid Earth (1978-2012) 113 (B5).

Dobler, C., Pappenberger, F., 2013. Global sensitivity analyses for a complex hydrological model applied in an alpine watershed. Hydrological Processes 27 (26), 3922-3940.

630

Dongarra, J., Faverge, M., Ltaief, H., Luszczek, P., 2014. Achieving numerical accuracy and high performance using recursive tile lu factorization with partial pivoting. Concurrency and Computation: Practice and Experience 26 (7), 1408-1431. 
Forrester, A. I., Bressloff, N. W., Keane, A. J., 2006. Optimization using surrogate models and partially converged computational fluid dynamics simulations. In: Proceedings of the Royal Society of London A: Mathematical, Physical and Engineering Sciences. Vol. 462. The Royal Society, pp. 21772204.

Forrester, A. I., Sóbester, A., Keane, A. J., 2007. Multi-fidelity optimization via surrogate modelling. In: Proceedings of the Royal Society of London A: Mathematical, Physical and Engineering Sciences. Vol. 463. The Royal Society, pp. 3251-3269.

$\mathrm{Fu}, \mathrm{R} ., 2013$. The effect of different rainfall information on sewer flow predictions. Master's thesis, ETH Zurich.

Giustolisi, O., Laucelli, D., 2005. Improving generalization of artificial neural networks in rainfallrunoff modelling. Hydrological Sciences Journal 50 (3).

Goffe, W. L., Ferrier, G. D., Rogers, J., 1992. Simann: Fortran module to perform global optimization of statistical functions with simulated annealing.

Gramacy, R. B., Lian, H., 2012. Gaussian process single-index models as emulators for computer experiments. Technometrics 54 (1), 30-41.

Gugercin, S., Antoulas, A. C., 2004. A survey of model reduction by balanced truncation and some new results. International Journal of Control 77 (8), $748-766$.

Haag, I., 2006. A basic water quality model for the river neckar: part 1-model development, parameter sensitivity and identifiability, calibration and validation. Acta hydrochimica et hydrobiologica 34 (6), 533-548.

Higdon, D., Gattiker, J., Williams, B., Rightley, M., 2008. Computer model calibration using high-dimensional output. Journal of the American Statistical Association 103 (482).

Huber, W., of Guelph. School of Engineering, U., Dickinson, R., Aldrich, J., Roesner, L., Barnwell, T., Laboratory, E. R., Laboratory, C. E. R., EPA, U., 1988. The USEPA SWMM4 Stormwater Management Model: Version 4 User's Manual. University of Guelph, School of Engineering.

URL http://books . google.ch/books?id=8UewnQEACAAJ

Kennedy, M. C., O'Hagan, A., 2001. Bayesian calibration of computer models. Journal of the Royal Statistical Society. Series B (Statistical Methodology) 63 (3), 425-464.

Leary, S. J., Bhaskar, A., Keane, A. J., 2003. A knowledge-based approach to response surface modelling in multifidelity optimization. Journal of Global Optimization 26 (3), 297-319. 
Legendre, M., Schmidt, A., Moussaoui, S., Lammers, U., 2013. Solving systems of linear equations by gpu-based matrix factorization in a science ground segment. Astronomy and Computing 34 (0), 58 - 64 .

Liu, F., West, M., 2009. A dynamic modelling strategy for Bayesian computer model emulation. Bayesian analysis 4, 393-412.

Lunati, I., Jenny, P., 2008. Multiscale finite-volume method for density-driven flow in porous media. Computational Geosciences 12 (3), 337-350.

Machac, D., Reichert, P., Albert, C., 2015. Emulation of dynamic simulators with application to hydrology. Journal of Computational PhysicsIn review.

Mancipe-Munoz, N. A., Buchberger, S. G., Suidan, M. T., Lu, T., 2014. Calibration of rainfall-runoff model in urban watersheds for stormwater management assessment. Journal of Water Resources Planning and Management 140 (6), 05014001 .

McKay, M., Beckman, R., Conover, W., May 1979. Comparison the three methods for selecting values of input variable in the analysis of output from a computer code. Technometrics; (United States) 21:2.

Muleta, M. K., Boulos, P. F., 2008. Analysis and calibration of rdii and design of sewer collection systems. In: The World Environmental \& Water Resources Congress. Vol. 316. p. 642.

O'Hagan, A., 1992. Some bayesian numerical analysis. Bayesian statistics 4 (345$363), 4-2$.

O'Hagan, A., 2006. Bayesian analysis of computer code outputs: A tutorial. Reliability Engineering and System Safety 91, 1290-1300.

Razavi, S., Tolson, B. A., Burn, D. H., 2012. Review of surrogate modeling in water resources. Water Resources Research 48 (7).

Reichert, P., White, G., Bayarri, M. J., Pitman, E. B., Apr. 2011. Mechanismbased emulation of dynamic simulation models: Concept and application in hydrology. Computational Statistics \& Data Analysis 55 (4), 1638-1655.

Schütze, M., Campisano, A., Colas, H., Schilling, W., Vanrolleghem, P. A., 2004. Real time control of urban wastewater systemswhere do we stand today? Journal of hydrology 299 (3), 335-348.

Spiller, E. T., Bayarri, M., Berger, J. O., Calder, E. S., Patra, A. K., Pitman, E. B., Wolpert, R. L., 2014. Automating emulator construction for geophysical hazard maps. SIAM/ASA Journal on Uncertainty Quantification 2 (1), 126152.

Stieglitz, M., Hobbie, J., Giblin, A., Kling, G., 1999. Hydrologic modeling of an arctic tundra watershed: toward pan-arctic predictions. Journal of Geophysical Research. D. Atmospheres 104, 27. 
Sudret, B., 2008. Global sensitivity analysis using polynomial chaos expansions. in Dependability.

Vanrolleghem, P., Benedetti, L., Meirlaen, J., 2005. Modelling and real-time control of the integrated urban wastewater system. Environmental Modelling \& Software 20 (4), 427 - 442, vulnerability of Water Quality in Intensively Developing Urban Watersheds.

Zurada, J. M., 1992. Introduction to artificial neural systems. West St. Paul.

\section{List of symbols}

$\begin{array}{ll}\text { simulator } & \\ A & \text { area of a subcatchment } \\ f & \text { model equations } \\ \mathbf{g} & \text { observation function } \\ n_{\text {surf }} & \text { Manning-Strickler coefficient of a subcatchment } \\ n_{\text {net }} & \text { Manning-Strickler coefficient of a channel } \\ N_{t} & \text { length of time series } \\ r & \text { \% of impervious area } \\ s & \text { slope of a subcatchment } \\ t & \text { time variable } \\ w & \text { width of an overland flow path } \\ x & \text { spatial variable } \\ X & \text { state space } \\ y & \text { output of the full model } \\ \boldsymbol{\theta} & \text { vector of parameter factors } \\ \boldsymbol{\Xi} & \text { state of the system }\end{array}$




\begin{tabular}{|c|c|}
\hline$a, b$ & indices of a particular linear reservoir \\
\hline$c$ & celerity of a wave \\
\hline $\mathbf{C}$ & covariance matrix of the white noise \\
\hline $\mathbf{F}$ & transformation matrix \\
\hline$\widetilde{\mathbf{F}}$ & transformation matrix for the coupled system \\
\hline$h$ & water height (state variable) \\
\hline$H$ & observation operator \\
\hline$k_{n e t}$ & estimated parameter of the sub-surface module \\
\hline$k_{\text {surf }}$ & estimated parameter of the surface module \\
\hline$l$ & Euclidean distance \\
\hline $\mathcal{L}$ & likelihood function \\
\hline$m$ & dimension of the simplified state space \\
\hline$n$ & number of design data sets \\
\hline$p$ & precipitation intensity \\
\hline $\mathbf{P}$ & input vector \\
\hline$\widetilde{\mathbf{P}}$ & input vector for the coupled system \\
\hline$Q$ & flow \\
\hline $\mathbf{R}$ & relative amplitude \\
\hline$S$ & storage (state variable) \\
\hline$X^{\prime}$ & simplified state space \\
\hline$\overline{\mathbf{y}}$ & output of the emulator \\
\hline $\mathbf{z}$ & mean of the unconditioned system \\
\hline$\alpha, \beta$ & indices denoting replicas \\
\hline$\gamma$ & correlation length \\
\hline$\eta$ & white noise \\
\hline$\theta^{\prime}$ & parameter vector of the auxiliary parameters \\
\hline$\widetilde{\boldsymbol{\theta}}$ & vector of parameter factors of the coupled system \\
\hline$\kappa$ & release coefficient of the surface reservoir \\
\hline$\lambda$ & release coefficient of the sewer reservoir \\
\hline$\mu$ & estimated coefficient for an observation of the water level in a manhole \\
\hline$\xi$ & state vector in the simplified state space \\
\hline$\widetilde{\xi}$ & state vector of the coupled system \\
\hline$\sigma$ & amplitude of the white noise \\
\hline $\bar{\Sigma}$ & covariance matrix of the unconditioned system \\
\hline$\Sigma$ & covariance matrix of the emulator \\
\hline
\end{tabular}

